\title{
Mitochondria-related TFAM gene variants and their effects on patients with cervical cancer
}

\author{
IEVA GOLUBICKAITE ${ }^{1}$, RASA UGENSKIENE $^{1,2}$, JUSTE CEPAITE ${ }^{2}$, EGLE ZILIENE ${ }^{2}$, \\ ARTURAS INCIURA $^{2}$, LINA POSKIENE ${ }^{3}$ and ELONA JUOZAITYTE ${ }^{2}$ \\ ${ }^{1}$ Department of Genetics and Molecular Medicine, ${ }^{2}$ Institute of Oncology, ${ }^{3}$ Department of Pathological Anatomy, \\ Lithuanian University of Health Sciences, 44307 Kaunas, Lithuania
}

Received July 23, 2021; Accepted October 13, 2021

DOI: $10.3892 /$ br.2021.1482

\begin{abstract}
Cervical cancer is the fourth most common type of cancer in women worldwide, with high incidence and mortality rates, particularly in developing countries. There are human papillomavirus vaccines and cytological screening programs available; however, there are no molecular markers that would aid the prognosis of the course of the disease or prediction of the outcomes of the patients. The aim of the present study was to investigate the associations between single nucleotide polymorphisms (SNPs) of the mitochondrial transcription factor A (TFAM) gene (rs11006132, rs11006129, rs1937, rs16912174, rs16912202 and rs3900887), and the clinical parameters and tumor phenotype of patients with cervical cancer. DNA isolated from patients with cervical cancer $(n=172)$ was used for genotyping using Real-Time PCR using TaqMan probes. It was revealed that the TFAM rs3900887 TT and AT genotypes were associated with a lower risk of developing larger tumors. The results showed an association between the rs3900887 SNP and tumor phenotype, indicating TFAM rs3900887 as a potential biomarker for tumor size in cervical cancer.
\end{abstract}

\section{Introduction}

Cervical cancer is frequently diagnosed in women and, with a $6.5 \%$ incidence rate, ranks as the fourth most common type of cancer globally. Furthermore, it also ranks fourth in mortality statistics, with a rate of $7.7 \%$ (1). Cervical cancer incidence and mortality rates are higher in developing than in developed countries. The vast majority of the highest cervical cancer rates are observed in countries in sub-Saharan Africa, Melanesia, South America and South-Eastern Asia (1). Previous studies have demonstrated that cervical cancer is a disease caused by multiple interacting factors, and thus, human papillomavirus (HPV) is

Correspondence to: Dr Ieva Golubickaite, Department of Genetics and Molecular Medicine, Lithuanian University of Health Sciences, A. Mickeviciaus str. 9, 44307 Kaunas, Lithuania

E-mail: ieva.golubickaite@1smuni.lt

Key words: mitochondrial transcription factor A, cervical cancer, single nucleotide polymorphism, tumor phenotype a necessary but not sufficient cause of cervical cancer occurrence (2). Infections, such as human immunodeficiency virus, and environmental factors, such as smoking or long-term use of oral contraceptives, are important co-factors (3). Cervical cancer is considered highly preventable, and HPV vaccination and screening programs are widely used for preventative purposes $(4,5)$. Countries that successfully implement these measures have lower cervical cancer rates. For example, in the United States, cervical cancer incidence rates declined $1.9 \%$ per year between 2007 and 2012 (6). However, numerous lower-income countries have not fulfilled mass screening programs, and the cervical cancer incidence and mortality rates remain high (7). Therefore, to reduce the gap between developing and developed countries, and help diagnose cervical cancer during the early stages, predict the course of the disease and predict the outcome, more accessible screening options are required.

Mitochondria are essential and complex organelles involved in cellular processes, such as energy generation through oxidative phosphorylation, apoptosis and metabolism. Therefore, mitochondria are considered to serve an essential role in carcinogenesis (8). Mitochondrial transcription factor A $(T F A M)$ is a nuclear-encoded protein, which serves an important role in mitochondria, since it is required for the transcription and replication of mitochondrial DNA $(9,10)$. TFAM expression has been reported to be altered and associated with breast and lung cancer (11-13), and polymorphisms in the TFAM gene have been reported to be associated with prostate (14), colorectal (15), breast (16), uterine, ovary and cervical cancer (17). Numerous studies have investigated the associations between various single nucleotide polymorphisms (SNPs) and the risk of cervical cancer; however, there are not enough studies analyzing mitochondria-related SNPs and the associations with the clinical/pathological characteristics of the patients or the morphological characteristics of their tumors (18). In the present study, genotyping analysis was used to investigate TFAM SNP distribution in a cervical cancer cohort, and to explore their potential as biomarkers for the tumor phenotype or outcomes of patients.

\section{Materials and methods}

Study subjects. The study population consisted of 172 female patients who were diagnosed with cervical cancer. All patients were Lithuanians with a median age of 56 years (age range, 22-83 years). Patients were included in the present study if they 
matched all of the following criteria: Cervical cancer was diagnosed as a primary disease, the patient signed a consent form, a blood sample was taken at the time of the diagnosis and clinical information was available. In our previous study, the same patient group was analyzed focusing on the polymorphisms of a different gene (DNA polymerase $\gamma$, catalytic subunit) and their associations with cervical cancer (18). The research was conducted at the Institute of Oncology, Lithuanian University of Health Sciences (Kaunas, Lithuania) between 2015 January and 2021 May. The present study was performed in accordance to the guidelines of the Declaration of Helsinki (19) and was approved by the Kaunas Regional Biomedical Research Ethics Committee (approval nos. BE-2-10 and P1-BE-2-10/2014; Kaunas, Lithuania). Clinical data were gathered retrospectively from medical records.

Tumor grading. At present no particular grading system has achieved universal acceptance, and grading of cervical carcinomas remains of uncertain clinical value. In the present study histopathological grading $(\mathrm{G})$ was based on the degree of cell and tissue atypia and mitotic activity. The definitions of the G categories apply to all carcinomas, these are: GX, grade of differentiation cannot be assessed; G1, well differentiated; G2, moderately differentiated; and G3, poorly differentiated or undifferentiated (20).

Genotyping. DNA was isolated from EDTA-preserved peripheral blood using a GeneJet Genomic DNA purification kit (cat. no. K0721; Thermo Fisher Scientific, Inc.). SNPs (rs11006132, rs11006129, rs1937, rs16912174, rs16912202 and rs3900887) in the TFAM gene were determined using TaqMan probe SNP Genotyping assays (cat. no. 4351379; Thermo Fisher Scientific, Inc.) and a QuantStudio 3 Real-Time PCR system (cat. no. A28137; Thermo Fisher Scientific, Inc.). The reaction mixture contained $15 \mathrm{ng}$ purified DNA sample, $6.125 \mu 1$ TaqMan Universal MasterMix (cat. no. 4304437; Thermo Fisher Scientific, Inc.), $0.625 \mu \mathrm{l}$ TaqMan SNP Genotyping assay and nuclease-free water to reach a total volume of $12 \mu \mathrm{l}$. Nuclease-free water was used as a no-template control for every plate. The standard genotyping PCR program: Pre-read step at $60^{\circ} \mathrm{C}$ for $30 \mathrm{sec}, 95^{\circ} \mathrm{C}$ for $10 \mathrm{~min}$; followed by 40 cycles of $95^{\circ} \mathrm{C}$ for $15 \mathrm{sec}$ and $60^{\circ} \mathrm{C}$ for $1 \mathrm{~min}$; with a final post-read step at $60^{\circ} \mathrm{C}$ for $30 \mathrm{sec}$, was used to determine the genotype, and this relied on VIC and FAM fluorescence intensity.

Statistical analysis. Associations between all TFAM polymorphisms included in the present study (rs11006132, rs11006129, rs1937, rs16912174, rs16912202 and rs3900887) and clinical data, pathological tumor size (T), tumor differentiation grade $(\mathrm{G})$, lymph node status $(\mathrm{N})$, distant metastasis $(\mathrm{M})$, histological tumor type, progression and death, were analyzed. Additionally, patients were divided into two groups according to median age at diagnosis ( $<56$ and $\geq 56$ years old) and pathological tumor size [small tumors $(\mathrm{T} 1+\mathrm{T} 2 ; \mathrm{n}=110)$ and larger tumors $(\mathrm{T} 3+\mathrm{T} 4 ; \mathrm{n}=62)]$. Furthermore, patients were divided into groups based on tumor differentiation grade; the first group included patients with well-differentiated and moderately differentiated tumors $(\mathrm{G} 1+\mathrm{G} 2)$, and the second group included patients with poorly differentiated tumors (G3).

SPSS version 22 software (IBM Corp.) was used for association analysis. Associations between genotype and clinical data were evaluated using a Pearson's $\chi^{2}$ test (rs11006132,
Table I. Demographic and clinicopathological characteristics of the patients.

\begin{tabular}{lc}
\hline Characteristics & Frequency, $\mathrm{n}(\%)$ \\
\hline Stage & \\
I & $16(9.3)$ \\
II & $60(34.9)$ \\
III & $83(48.3)$ \\
IV & $13(7.6)$ \\
Tumor size & \\
T1 & $26(15.1)$ \\
T2 & $84(48.8)$ \\
T3 & $55(32)$ \\
T4 & $7(4.1)$ \\
Lymph node status & \\
Negative & $95(55.2)$ \\
Positive & $77(44.8)$ \\
Distant metastasis & \\
Absent & $162(94.2)$ \\
Present & $10(5.8)$ \\
Differentiation grade & \\
G1 & $13(7.6)$ \\
G2 & $113(65.7)$ \\
G3 & $44(25.6)$ \\
Missing & $2(1.2)$ \\
Survival status & $132(76.7)$ \\
Alive & $40(23.3)$ \\
Deceased & $123(71.5)$ \\
Fact of progression & $49(28.5)$ \\
Absent & \\
Present & \\
\hline
\end{tabular}

a2 data points missing due to lack of patients' clinical information.

rs11006129, rs1937 and rs3900887) or Fisher's Exact (rs16912174 and rs16912202) tests and binary logistic regression. Univariate logistic regression analysis adjusted for age at diagnosis (Model A) and multivariate logistic regression analysis with additional confounding factor N (Model B), were used. Survival analysis was performed using Kaplan-Meier analysis and the difference between survival curves was analyzed using a log-rank test. $\mathrm{P}<0.05$ was considered to indicate a statistically significant difference.

\section{Results}

Distribution of clinical characteristics. The present study included 172 patients with cervical cancer with a median age of 56 years (age range, 22-83 years). The majority of the patients had squamous cell carcinoma $(n=157)$, whereas adenocarcinoma $(n=9)$ or mucinous adenocarcinoma $(n=6)$ were less frequent. The tumor differentiation grade was mostly G2 $(n=113)$. The majority of the patients did not have distant metastasis $(n=162)$ or affected regional lymph nodes $(\mathrm{n}=95)$. Additional information on the clinical characteristics are provided in Table I. 

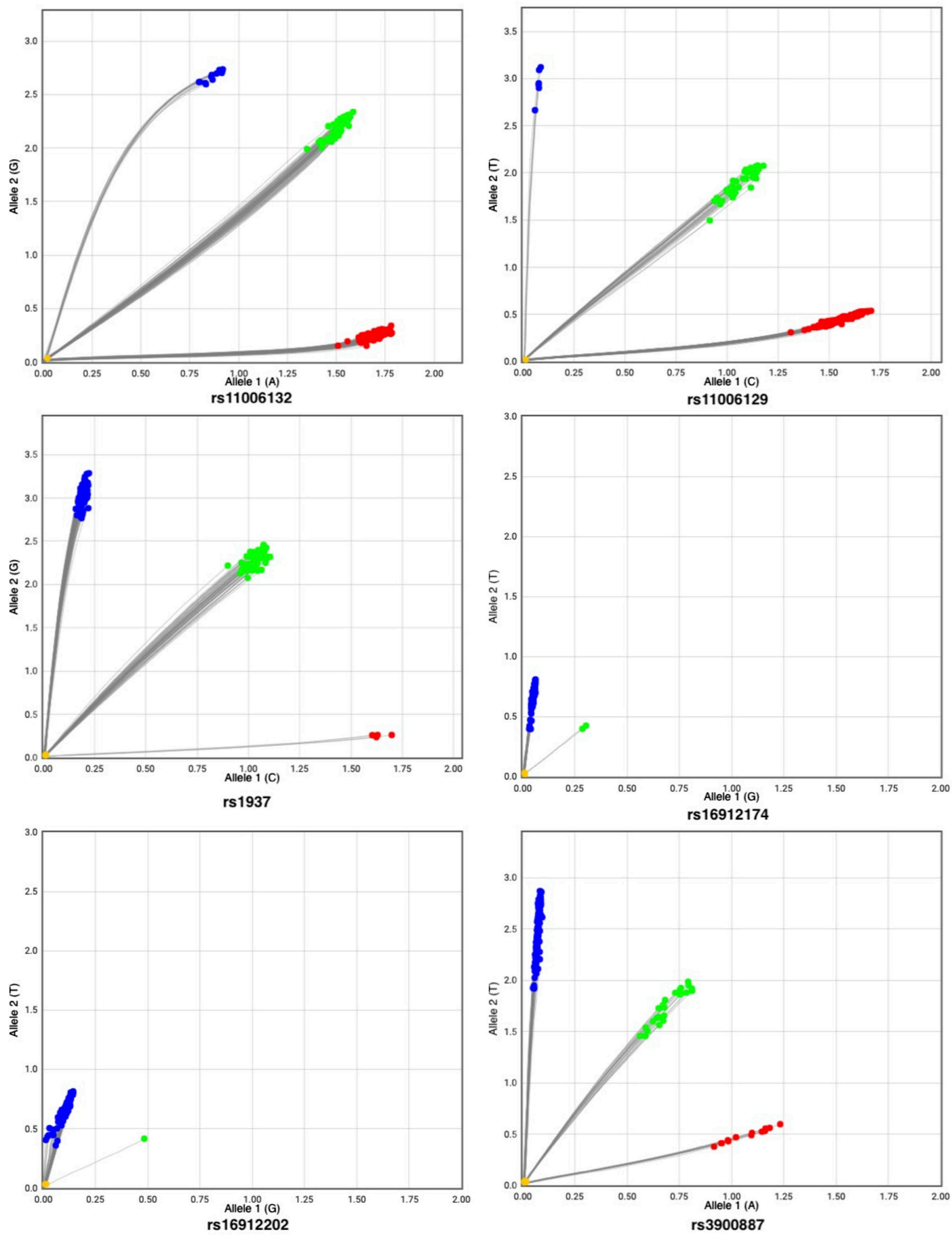

Figure 1. Distribution of TFAM genotypes. Allelic discrimination plots representing the TFAM rs11006132, rs11006129, rs1937, rs16912174, rs16912202, rs3900887 genotypes in the cervical cancer cohort. The x-axis represents Allele 1 labeled with VIC dye, and the y-axis represents Allele 2 labeled with FAM dye. The colored dots represent different genotypes called according to VIC and FAM fluorescence intensities. Red dots represents the homozygous Allele 1 genotype, blue dots represents the homozygous Allele 2 genotype, and the green dots represents the heterozygous genotype. The no-template control is marked as a yellow dot at the bottom left corner of the plot. TFAM, TFAM, mitochondrial transcription factor A.

Distribution of genotypes. The distributions of all genotypes in the present cohort were estimated using the Hardy-Weinberg equilibrium (HWE), and it was revealed that rs11006132, rs11006129, rs1937, rs16912174 and rs16912202 SNPs were in HWE, whereas rs3900887 was not. There were 13 missing data points from rs3900887 genotyping due to amplification failure. The distribution of TFAM genotypes is shown in Fig. 1 and Table II. 


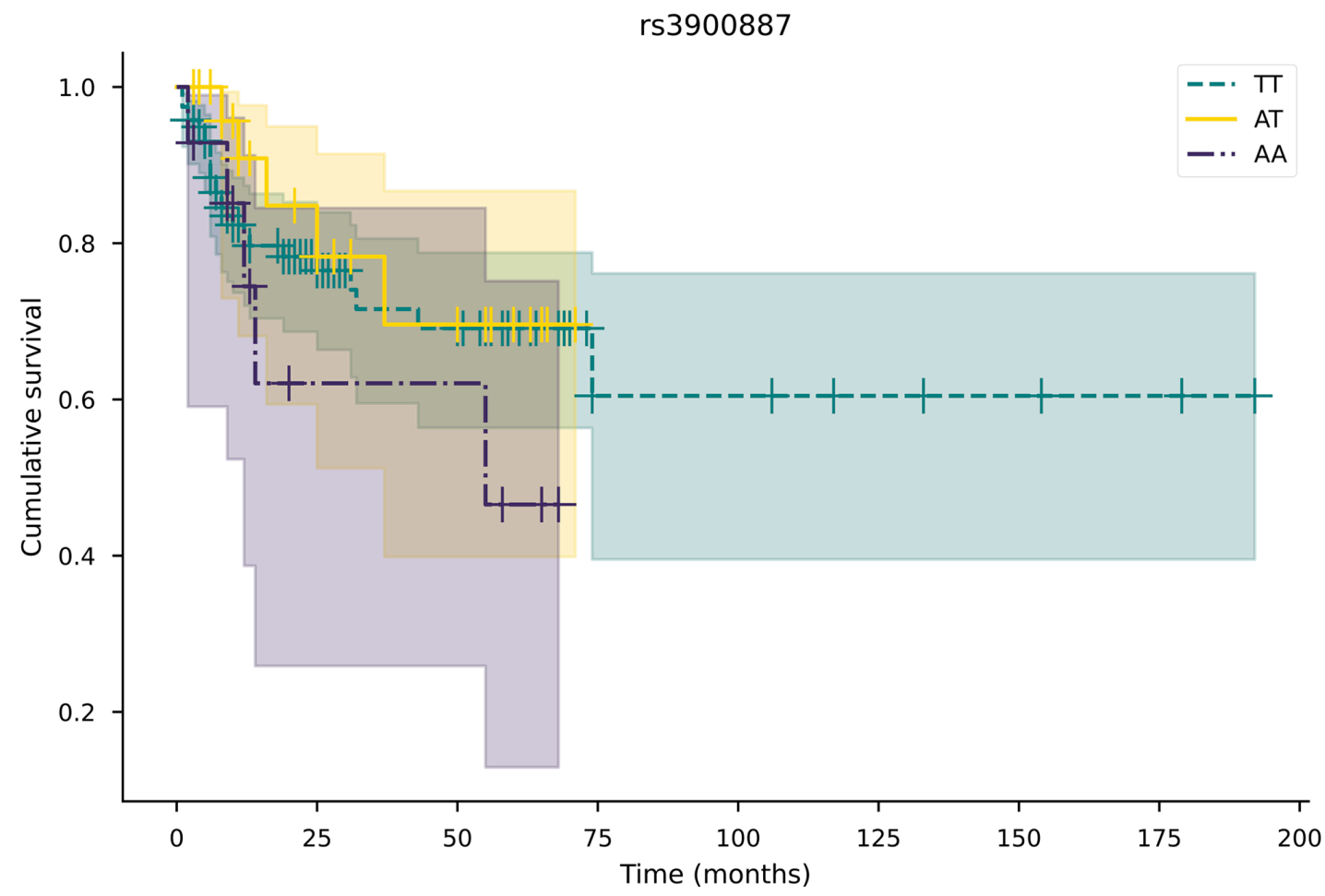

Figure 2. Overall survival of the patients with cervical cancer with different TFAM rs3900887 genotypes. Kaplan-Meier survival curves for overall survival comparing different TFAM rs3900887 genotypes: TT vs. AT ( $\mathrm{P}=0.548)$, TT vs. AA $(\mathrm{P}=0.357)$ and AT vs. AA $(\mathrm{P}=0.195)$ in the cervical cancer cohort. The $\mathrm{x}$-axis represents the overall survival of the patients in months, and the y-axis represents cumulative survival. Vertical lines indicate censored cases. The different colors represent different genotypes; the shadow of the same color represents the respective $95 \%$ confidence interval.

Table II. Genotype and allele frequencies of the analyzed TFAM polymorphisms in the study population.

\begin{tabular}{|c|c|c|c|}
\hline TFAM & $\begin{array}{l}\text { Patient } \\
\text { count }\end{array}$ & $\begin{array}{l}\text { Genotype, } \\
\text { frequency }\end{array}$ & $\begin{array}{l}\text { Allele, } \\
\text { frequency }\end{array}$ \\
\hline \multirow[t]{3}{*}{ rs11006132 } & 90 & AA, 0.52 & A, 0.72 \\
\hline & 68 & $\mathrm{AG}, 0.4$ & $\mathrm{G}, 0.28$ \\
\hline & 14 & GG, 0.08 & \\
\hline \multirow[t]{3}{*}{ rs11006129 } & 130 & $\mathrm{CC}, 0.76$ & $\mathrm{C}, 0.86$ \\
\hline & 36 & CT, 0.21 & $\mathrm{~T}, 0.14$ \\
\hline & 6 & TT, 0.03 & \\
\hline \multirow[t]{3}{*}{ rs1937 } & 115 & $\mathrm{GG}, 0.67$ & $\mathrm{G}, 0.82$ \\
\hline & 53 & $\mathrm{CG}, 0.31$ & C, 0.18 \\
\hline & 4 & $\mathrm{CC}, 0.02$ & \\
\hline \multirow[t]{2}{*}{ rs16912174 } & 170 & TT, 0.99 & $\mathrm{~T}, 0.99$ \\
\hline & 2 & GT, 0.01 & $\mathrm{G}, 0.01$ \\
\hline \multirow[t]{2}{*}{ rs16912202 } & 171 & TT, 0.99 & $\mathrm{~T}, 1.00$ \\
\hline & 1 & GT, 0.01 & $\mathrm{G}, 0.00$ \\
\hline \multirow[t]{3}{*}{ rs3900887 } & 118 & $\mathrm{TT}, 0.74$ & $\mathrm{~T}, 0.83$ \\
\hline & 27 & AT, 0.17 & A, 0.17 \\
\hline & 14 & AA, 0.09 & \\
\hline
\end{tabular}

TFAM, mitochondrial transcription factor A.

Association analysis. Associations between TFAM polymorphisms and clinical data were evaluated using Pearson's $\chi^{2}$ or Fisher's exact tests and are presented in Table III.
The present study revealed that rs3900887 was associated with grouped tumor size $\mathrm{T} 1+\mathrm{T} 2$ vs. $\mathrm{T} 3+\mathrm{T} 4(\mathrm{P}=0.006)$. However, no associations with age group $(\mathrm{P}=0.905)$, lymph node status $(\mathrm{P}=0.468)$, distant metastasis $(\mathrm{P}=0.166)$, differentiation grade $(\mathrm{P}=0.898)$, adenocarcinoma $(\mathrm{P}=0.376)$, squamous cell carcinoma $(\mathrm{P}=0.480)$, stage $(\mathrm{P}=0.065)$, progression $(\mathrm{P}=0.258)$ or death $(\mathrm{P}=0.457)$ were observed. To further evaluate the associations amongst rs3900887 and clinical data, logistic regression analysis was performed. It was revealed that rs3900887 remained significantly associated with grouped tumor size in logistic regression analysis of genotype and allele models. Patients with the rs3900887 TT [odds ratio $(\mathrm{OR})=0.328 ; 95 \% \mathrm{CI}, 0.127-0.849 ; \mathrm{P}=0.022$ ] or AT (OR=0.091; 95\% CI, 0.023-0.367; $\mathrm{P}=0.001)$ genotypes were less likely to have larger tumors than those with the AA genotype. Univariate and multivariate logistic regression analyses are presented in Table IV.

No statistically significant associations of rs11006132, rs11006129, rs1937, rs16912174 and rs16912202 with clinical data were observed.

Survival analysis. Kaplan-Meier survival analysis was used to determine the association between rs3900887 and overall survival; however, it was not statistically significant: TT vs. AT $(\mathrm{P}=0.548)$, TT vs. AA $(\mathrm{P}=0.357)$, AT vs. AA $(\mathrm{P}=0.195)$. The Kaplan-Meier survival plot is shown in Fig. 2.

\section{Discussion}

Despite extensive prevention and vaccination programs, cervical cancer remains the fourth most common cancer in 
Table III. Associations between mitochondrial transcription factor A polymorphisms and clinical data.

\begin{tabular}{|c|c|c|c|c|c|c|}
\hline Variable & rs 11006132 & rs 11006129 & rs1937 & rs16912174 & rs16912202 & rs3900887 \\
\hline $\mathrm{T}(\mathrm{T} 1+\mathrm{T} 2$ vs. $\mathrm{T} 3+\mathrm{T} 4$ & 0.789 & 0.105 & 0.309 & 0.408 & 0.640 & 0.006 \\
\hline N (negative vs. positive) & 0.979 & 0.893 & 0.954 & 0.696 & 0.448 & 0.468 \\
\hline M (negative vs. positive) & 0.427 & 0.382 & 0.211 & 0.887 & 0.058 & 0.166 \\
\hline Differentiation grade (G1 + G2 vs. G3) & 0.417 & 0.910 & 0.439 & 0.452 & 0.741 & 0.898 \\
\hline Stage (I + II vs. III + IV) & 0.775 & 0.225 & 0.966 & 0.690 & 0.558 & 0.065 \\
\hline Survival status (alive vs. deceased) & 0.565 & 0.833 & 0.661 & 0.588 & 0.767 & 0.457 \\
\hline Fact of progression & 0.310 & 0.209 & 0.986 & 0.510 & 0.715 & 0.258 \\
\hline Age group $(<56$ vs. $\geq 56)$ & 0.249 & 0.546 & 0.605 & 0.254 & 0.494 & 0.905 \\
\hline Adenocarcinoma & 0.472 & 0.150 & 0.734 & 0.898 & 0.948 & 0.376 \\
\hline Squamous cell carcinoma & 0.328 & 0.615 & 0.812 & 0.833 & 0.913 & 0.480 \\
\hline
\end{tabular}

T, pathological tumor size; $\mathrm{N}$, lymph node status; $\mathrm{M}$, distant metastasis.

Table IV. Univariate and multivariate logistic regression analysis of the associations of mitochondrial transcription factor A rs3900887 genotypes, alleles and clinical data with potential confounding factors.

\begin{tabular}{|c|c|c|c|c|c|c|c|c|}
\hline \multirow[b]{2}{*}{$\begin{array}{l}\text { Single nucleotide } \\
\text { polymorphism }\end{array}$} & \multirow[b]{2}{*}{ Parameter } & \multirow[b]{2}{*}{ Covariates } & \multicolumn{3}{|c|}{ Model A } & \multicolumn{3}{|c|}{ Model B } \\
\hline & & & $\begin{array}{l}\text { Odds } \\
\text { ratio }\end{array}$ & $\begin{array}{c}95 \% \text { confidence } \\
\text { interval }\end{array}$ & P-value & $\begin{array}{l}\text { Odds } \\
\text { ratio }\end{array}$ & $\begin{array}{c}95 \% \text { confidence } \\
\text { interval }\end{array}$ & P-value \\
\hline \multirow[t]{4}{*}{ rs3900887 } & Tumor size & TT vs. AA & 0.328 & $0.127-0849$ & $0.022^{\mathrm{a}}$ & 0.350 & $0.097-1.261$ & 0.108 \\
\hline & $(\mathrm{T} 1+\mathrm{T} 2 \mathrm{vs}$. & AT vs. AA & 0.091 & $0.023-0.367$ & $0.001^{\mathrm{b}}$ & 0.093 & $0.018-0.490$ & $0.005^{\mathrm{b}}$ \\
\hline & $\mathrm{T} 3+\mathrm{T} 4)$ & Age & 1.011 & $0.996-1.028$ & 0.156 & 1.038 & $1.008-1.070$ & $0.013^{\mathrm{a}}$ \\
\hline & & $\begin{array}{l}\text { Lymph node status } \\
\text { (negative vs. positive) }\end{array}$ & - & - & - & 7.242 & $3.199-16.395$ & $<0.0001^{\mathrm{c}}$ \\
\hline \multirow[t]{3}{*}{ rs3900887 T allele } & $\begin{array}{l}\text { Tumor size } \\
(\mathrm{T} 1+\mathrm{T} 2 \text { vs. } \\
\text { T3 + T4) }\end{array}$ & Carrier vs. non-carrier & 0.277 & 0.109-0.709 & $0.007 \mathrm{~b}$ & 0.286 & $0.080-1.020$ & 0.054 \\
\hline & & Age & 1.011 & $0.995-1.027$ & 0.168 & 1.037 & $1.007-1.068$ & $0.014^{\mathrm{a}}$ \\
\hline & & $\begin{array}{l}\text { Lymph node status } \\
\text { (negative vs. positive) }\end{array}$ & - & - & - & 7.105 & $3.195-15.799$ & $<0.0001^{\mathrm{c}}$ \\
\hline
\end{tabular}

${ }^{\mathrm{a}} \mathrm{P}<0.05,{ }^{\mathrm{b}} \mathrm{P}<0.01,{ }^{\mathrm{c}} \mathrm{P}<0.001$.

women globally and has the fourth highest mortality rate amongst all types of cancer (1). In more developed countries with better established screening and vaccination programs, the incidence rates of cervical cancer have been declining each year (6); however, it is considerably more common in developing countries (1).

Mitochondria are organelles responsible for energy generation, apoptosis and metabolism, and serve an essential role in cancer development (8). The TFAM gene encodes mitochondrial transcription factor, a protein encoded in nuclear DNA that is responsible for the transcription and translation of mitochondrial DNA $(9,10)$. Research has revealed that SNPs in the TFAM gene are associated with various types of cancer (14-16), including cervical cancer (17). Numerous studies have assessed the association between the risk of developing cervical cancer and SNPs; however, few studies have investigated the possible associations between SNPs and clinical and morphological cervical tumor characteristics. In the present study, the associations of the rs11006132, rs11006129, rs1937, rs16912174, rs16912202 and rs3900887 polymorphisms in the TFAM gene with cervical tumor clinical and morphological characteristics, including tumor histological type, stage, size, differentiation grade, regional lymph node involvement, distant metastases, progression and survival status, were analyzed.

It was revealed that the TFAM rs3900887 polymorphism was associated with tumor characteristics. Logistic regression analysis indicated that patients with TT and TA genotypes had a lower risk of possessing larger tumors than patients with the AA genotype. rs3900887 is located in an intron of the TFAM gene and it may affect splicing. However, it is also reported to be benign by VarSome (21) and Franklin (22) databases. The imum allele frequency (MAF) of this gene in 
the 1,000 Genome project was 0.11 , and a similar MAF $(0.17)$ was calculated in the present study. Our previous study identified several associations between this polymorphism and morphological characteristics of breast cancer. It was revealed that TT and TA genotypes were associated with an increased risk of positive lymph nodes, and TT genotype carriers also had an increased risk of positive estrogen receptors (ER) and lymphatic invasion compared with patients with the AA genotype (16). Additionally, there has been only one study investigating associations between this polymorphism and late-onset Alzheimer's disease, in which no significant associations were observed (23). Our recent study revealed significant associations between this SNP and tumor size, which remained significant after multivariate analysis. The results may indicate the role of the rs3900887 SNP in cervical tumor development, and thus, more in-depth studies are required to confirm its role in cervical tumor development.

Other SNPs in the present study (rs11006132, rs11006129, rs1937, rs16912174 and rs16912202) exhibited no significant associations with cervical tumor morphological parameters. The rs11006132 SNP is located in the 3' untranslated region of the gene. Our previous study indicated no associations between this SNP and morphological characteristics of breast cancer (16). There was also no observed association with aggressive prostate cancer (14). However, researchers have found a link between this SNP and the age at onset of Huntington's disease (24). rs11006129 is located in an intron of the gene. Our previous study demonstrated an association between this SNP and breast cancer. It was revealed that $\mathrm{T}$ allele carriers were less likely to be predisposed to ER-positive tumors and carriers of the $\mathrm{C}$ allele were less likely to develop tumors with vascular invasion than non-carriers of the respective allele (16). To the best of our knowledge, no other studies have investigated this SNP to date. rs1937 is a missense variant of the TFAM gene. Our previous study found no significant associations between this SNP and breast tumor parameters (16). Other studies have investigated the association between the rs1937 and early-onset myocardial infarction (25), late-onset Alzheimer's disease (26) and aggressive prostate cancer (14); however, no significant results were determined. One study has reported a link between the rs1937 polymorphism and cervical cancer development in women (27). The rs16912174 SNP is located in the 5 ' untranslated region of the gene. Our previous study revealed no significant association between this polymorphism and breast cancer (16). The rs16912202 polymorphism is located in the 3 ' untranslated region of the gene (c. $3236 \mathrm{~T}>\mathrm{G})$ and, therefore, it may interfere with mRNA stability or translation. Our previous study did not find any significant associations between this SNP and morphological characteristics of breast cancer (16). To the best of our knowledge, no other studies have been performed for this SNP in cervical cancer to date.

Despite the limitations of the present study, including the small sample size and small number of SNPs, it was possible to detect significant association, suggesting that the TFAM gene may serve a role in cervical cancer development. In this study the individual SNPs were analyzed as a pilot study to understand the impact of TFAM SNPs on cervical cancer. In future studies, larger cohorts will be employed to investigate the mitochondria-related SNPs in other genes, examining grouped SNPs and haplotypes, and their impact on cervical cancer. Additional studies should be performed on broader and more ethnically diverse cohorts of patients with cervical cancer to verify these results.

In conclusion, the results of the present study suggest that mitochondrial transcription factor A, encoded by the nuclear TFAM gene, is important in cervical cancer, and the rs 3900887 SNP may serve as a potential biomarker of tumor size.

\section{Acknowledgements}

Not applicable.

\section{Funding}

This research was funded by the Lithuanian University of Health Sciences (Kaunas, Lithuania).

\section{Availability of data and materials}

The datasets used and/or analyzed during the present study are available from the corresponding author on reasonable request.

\section{Authors' contributions}

IG, RU and EJ conceived the study. IG and JC developed the methodology. IG and JC performed the formal analysis. JC performed the experiments. RU, EZ, AI, LP and EJ curated the data. IG, RU and JC prepared the original draft of the manuscript. EZ, AI, LP and EJ reviewed and edited the manuscript. IG prepared the tables. RU and EJ supervised the study. All authors have read and approved the final manuscript. JC and IG confirm the authenticity of all the raw data.

\section{Ethics approval and consent to participate}

The present study was performed in accordance with the guidelines of the Declaration of Helsinki and approved by the Kaunas Regional Biomedical Research Ethics Committee (approval nos. BE-2-10 and P1-BE-2-10/2014). Informed written consent was obtained from all subjects involved in the present study.

\section{Patient consent for publication}

Not applicable.

\section{Competing interests}

The authors declare that they have no competing interests.

\section{References}

1. Sung H, Ferlay J, Siegel RL, Laversanne M, Soerjomataram I, Jemal A and Bray F: Global Cancer Statistics 2020: GLOBOCAN Estimates of Incidence and Mortality Worldwide for 36 Cancers in 185 Countries. CA Cancer J Clin 71: 209-249, 2021.

2. de Freitas AC, Gurgel APAD, Chagas BS, Coimbra EC and do Amaral CMM: Susceptibility to cervical cancer: An overview. Gynecol Oncol 126: 304-311, 2012.

3. Arbyn M, Weiderpass E, Bruni L, de Sanjosé S, Saraiya M, Ferlay $\mathrm{J}$ and Bray F: Estimates of incidence and mortality of cervical cancer in 2018: A worldwide analysis. Lancet Glob Health 8: e191-e203, 2020. 
4. Lei J, Ploner A, Elfström KM, Wang J, Roth A, Fang F, Sundström K, Dillner J and Sparén P: HPV Vaccination and the Risk of Invasive Cervical Cancer. N Engl J Med 383: 1340-1348, 2020.

5. Kessler TA: Cervical Cancer: Prevention and Early Detection. Semin Oncol Nurs 33: 172-183, 2017.

6. Benard VB, Thomas CC, King J, Massetti GM, Doria-Rose VP and Saraiya M; Centers for Disease Control and Prevention (CDC): Vital signs: Cervical cancer incidence, mortality, and screening - United States, 2007-2012. MMWR Morb Mortal Wkly Rep 63: 1004-1009, 2014.

7. Vaccarella S, Laversanne M, Ferlay J and Bray F: Cervical cancer in Africa, Latin America and the Caribbean and Asia: Regional inequalities and changing trends. Int J Cancer 141: 1997-2001, 2017.

8. van Gisbergen MW, Voets AM, Starmans MHW, de Coo IFM, Yadak R, Hoffmann RF, Boutros PC, Smeets HJM, Dubois L and Lambin P: How do changes in the mtDNA and mitochondrial dysfunction influence cancer and cancer therapy? Challenges, opportunities and models. Mutat Res Rev Mutat Res 764: 16-30, 2015.

9. Kang D, Kim SH and Hamasaki N: Mitochondrial transcription factor A (TFAM): Roles in maintenance of mtDNA and cellular functions. Mitochondrion 7: 39-44, 2007.

10. Hallberg BM and Larsson N-G: TFAM forces mtDNA to make a U-turn. Nat Struct Mol Biol 18: 1179-1181, 2011.

11. Gao W, Wu M-H, Wang N, Ying M-Z, Zhang Y-Y, Hua J, Chuan L and Wang Y-J: Mitochondrial transcription factor A contributes to cisplatin resistance in patients with estrogen receptor positive breast cancer. Mol Med Rep 14: 5304-5310, 2016.

12. Peng H, Yang M, Chen ZY, Chen P, Guan CX, Xiang XD, Cai S, Chen Y and Fang X: Expression and methylation of mitochondrial transcription factor a in chronic obstructive pulmonary disease patients with lung cancer. PLoS One 8: e82739, 2013.

13. Xie D, Wu X, Lan L, Shangguan F, Lin X, Chen F, Xu S, Zhang Y, Chen Z, Huang K, et al: Downregulation of TFAM inhibits the tumorigenesis of non-small cell lung cancer by activating ROS-mediated JNK/p38MAPK signaling and reducing cellular bioenergetics. Oncotarget 7: 11609-11624, 2016.

14. Granados JB, Méndez JP, Feria-Bernal G, García-García E, Tejeda ME, Rojano-Mejía D, Tapia A and Canto P: Association of a TFAM haplotype with aggressive prostate cancer in overweight or obese Mexican Mestizo men. Urol Oncol 35: 111. e9-111.e14, 2017.

15. Guo J, Zheng L, Liu W, Wang X, Wang Z, Wang Z, French AJ, Kang D, Chen L, Thibodeau SN, et al: Frequent truncating mutation of TFAM induces mitochondrial DNA depletion and apoptotic resistance in microsatellite-unstable colorectal cancer. Cancer Res 71: 2978-2987, 2011.

16. Golubickaite I, Ugenskiene R, Korobeinikova E, Gudaitiene J, Vaitiekus D, Poskiene L and Juozaityte E: The impact of mitochondria-related POLG and TFAM variants on breast cancer pathomorphological characteristics and patient outcomes. Biomarkers 26: 343-353, 2021.
17. Hu W, Ma S-L, Liu L-L, Zhu Y-H, Zeng T-T, Li Y and Guan X-Y: Impact of mitochondrial transcription factor A expression on the outcomes of ovarian, endometrial and cervical cancers. Am J Transl Res 12: 5343-5361, 2020.

18. Golubickaite I, Ugenskiene R, Ziliene E, Beniusyte J, Inciura A, Poskiene L and Juozaityte E: POLG Gene Variants in Cervical Cancer Patients and Their Associations with Clinical and Pathomorphological Tumor Characteristics. J Clin Med 10: 1838, 2021.

19. World Medical Association: World Medical Association Declaration of Helsinki: Ethical principles for medical research involving human subjects. JAMA 310: 2191-2194, 2013.

20. Kurman RJ, Carcangiu ML, Herrington S and Young RH (eds): WHO Classification of Tumours of Female Reproductive Organs. IARC, Lyon, 2014

21. Kopanos C, Tsiolkas V,Kouris A, Chapple CE, Albarca Aguilera M, Meyer R and Massouras A: VarSome: The human genomic variant search engine. Bioinformatics 35: 1978-1980, 2019.

22. Franklin by Genoox: The Future of Genomic Medicine. https://franklin.genoox.com/clinical-db/home. Accessed September 25, 2021.

23. Grupe A, Li Y, Rowland C, Nowotny P, Hinrichs AL, Smemo S, Kauwe JSK, Maxwell TJ, Cherny S, Doil L, et al: A scan of chromosome 10 identifies a novel locus showing strong association with late-onset Alzheimer disease. Am J Hum Genet 78: 78-88, 2006.

24. Taherzadeh-Fard E, Saft C, Akkad DA, Wieczorek S, Haghikia A, Chan A, Epplen JT and Arning L: PGC-1alpha downstream transcription factors NRF-1 and TFAM are genetic modifiers of Huntington disease. Mol Neurodegener 6: 32, 2011.

25. Palacín M, Alvarez V, Martín M, Díaz M, Corao AI, Alonso B, Díaz-Molina B,Lozano I, Avanzas P, Morís C, et al: Mitochondrial DNA and TFAM gene variation in early-onset myocardial infarction: Evidence for an association to haplogroup $\mathrm{H}$ Mitochondrion 11: 176-181, 2011.

26. Zhang Q, Yu J-T, Wang P, Chen W, Wu Z-C, Jiang H and Tan L: Mitochondrial transcription factor A (TFAM) polymorphisms and risk of late-onset Alzheimer's disease in Han Chinese. Brain Res 1368: 355-360, 2011.

27. Günther C, von Hadeln K, Müller-Thomsen T, Alberici A, Binetti G, Hock C, Nitsch RM, Stoppe G, Reiss J, Gal A, et al: Possible association of mitochondrial transcription factor A (TFAM) genotype with sporadic Alzheimer disease. Neurosci Lett 369: 219-223, 2004.

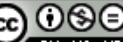

This work is licensed under a Creative Commons Attribution-NonCommercial-NoDerivatives 4.0 International (CC BY-NC-ND 4.0) License. 